\title{
Effects of Local Infiltration of Autologous Platelet Supernatant in Supraspinatus Tendinopathies
}

\author{
${ }^{1}$ Abhinav Gubba, ${ }^{2}$ Samir C Dwidmuthe, ${ }^{3}$ Anil Golhar, ${ }^{4}$ Nilesh Joshi
}

\begin{abstract}
Aim: The study was conducted to evaluate the therapeutic effects of platelet-rich plasma (PRP) injections in promoting healing of rotator cuff tendinopathies over short term by evaluating pain, range of motion (ROM) using Constant-Murley score (CMS).
\end{abstract}

Design: This prospective, cohort study was conducted in the Department of Orthopedics at a tertiary care teaching hospital between December 2013 and April 2015. Patients with supraspinatus tendinopathies (partial tear) based on either ultrasonography (USG) or magnetic resonance imaging (MRI) were included in the study. Patients with complete rotator cuff tear were excluded.

Materials and methods: Thirty consecutive patients with supraspinatus tendinopathies were administered subacromial injections of PRP. Platelet-rich plasma was made from $20 \mathrm{~mL}$ of patient's blood after subjecting it to two centrifugal spins and injected into the supraspinatus tendon. All patients were evaluated at the end of 3 months.

Results: Shoulder function was assessed by CMS in present study. Mean score at baseline was $33.87 \pm 5.91$. After 1 and 3 months, significant reduction in CMS was found with mean score of $27.80 \pm 5.17$ at 1 month $(p<0.0001)$ and $21.93 \pm 6.17$ at 3 months $(p<0.0001)$. There was a significant difference in score at 1 and 3 months $(p<0.0001)$. At 6,12 , and 17 months, the CMS was still significantly low but there was no difference in scores when compared with scores at 3 months.

Conclusion: Platelet-rich plasma injection for supraspinatus tendinopathies gives good results at the end of 3 months.

Keywords: Constant-Murley score, Infiltration, Platelet-rich plasma, Tendinitis.

How to cite this article: Gubba A, Dwidmuthe SC, Golhar A, Joshi N. Effects of Local Infiltration of Autologous Platelet Supernatant in Supraspinatus Tendinopathies. Int J Recent Surg Med Sci 2018;4(1):15-18.

Source of support: Nil

Conflict of interest: None

\section{INTRODUCTION}

Supraspinatus tendinopathy is a condition characterized by pain and decreased functionality of the affected

\footnotetext{
${ }^{1}$ Senior Resident, ${ }^{2}$ Associate Professor, ${ }^{3}$ Professor, ${ }^{4}$ Assistant Professor

${ }^{1-4}$ Department of Orthopedics, NKP Salve Institute of Medical Sciences, Nagpur, Maharashtra, India

Corresponding Author: Nilesh Joshi, Assistant Professor Department of Orthopedics, NKP Salve Institute of Medical Sciences, Nagpur, Maharashtra, India, e-mail: drjnilesh@ gmail.com
}

shoulder. It is often associated with shoulder impingement syndrome. ${ }^{1}$ The common belief is that impingement of the supraspinatus tendon leads to supraspinatus tendonitis (inflammation of the supraspinatus/rotator cuff tendon and/or the contiguous peritendinous soft tissues). ${ }^{2,3}$ Conventionally, this condition is treated with rest, pain therapy, physical therapy, and steroid injection. Of late, PRP infiltration has evolved as a promising modality in the treatment of various kinds of tendinopathies. ${ }^{4}$ Platelet-rich plasma owing to its high concentration of growth factors, such as platelet-derived growth factor, vascular endothelial growth factor, transforming growth factor $\beta$, and fibroblast growth factors has been shown to improve the quality of healing in tendinopathies. ${ }^{4}$ Our aim of this study has been to objectively evaluate the symptomatic and functional improvement following a single intralesional injection of PRP in cases of supraprinatus tendinopathies over the course of 17 months.

\section{MATERIALS AND METHODS}

This study was a prospective, cross-sectional study. The study was conducted in the Department of Orthopedics at a tertiary care teaching hospital, which caters to urban as well as rural population in central parts of India, between December 2013 and April 2015. Institutional ethics committee approved protocol of the study.

This study involved patients in outpatients and inpatients settings of orthopedic department. All patients who complained of pain in shoulder and/or restriction of shoulder movement were screened for the study. Patients with supraspinatus tendinopathies based on clinical findings and confirmed by USG consenting for the study were included. Magnetic resonance imaging was not done in all patients due to financial constraints.

Patients with history of fracture around shoulder joint, shoulder dislocation, and complete rotator cuff tear were excluded from the study. All relevant demographic details like age, gender, and occupation were noted. Baseline CMS was noted in each patient.

\section{PRP Preparation}

Patients were briefed about the procedure. Following steps were followed in PRP preparation: $20 \mathrm{cc}$ of blood was withdrawn from each patient's antecubital vein under all aseptic precautions. Citrate dextrose was used as anticoagulant. 
Initial centrifugation of blood was done with soft spin to separate red blood cells. After this, a second centrifugation was done with hard spin to concentrate the platelets; lower one-third contained PRP and upper one-third was removed, as it was platelet-poor plasma.

The lateral edge of the acromion on affected side is located with thumb. It is then passed along the edge anteriorly where bony prominence is felt. Then thumb is slid downward in front for quarter of an inch. The groove is felt where the distal end of the tendon lies.

Freshly prepared PRP was taken in syringe with needle no. 24 and around 2 to $3 \mathrm{~mL}$ of PRP was injected at the site of tendon. After single injection of PRP patients were followed up for a minimum of 3 months. At baseline and $1,3,6,12$, and 17 months, patients were assessed for pain and movement on affected site with the help of CMS.

\section{Constant-Murley Score}

The CMS is one of the first shoulder score systems developed and is considered the most commonly used scoring system for evaluation of various disorders of the shoulder. ${ }^{5}$ This score assesses subjective and objective shoulder function with respect to following parameters.

- A: Pain

- B: Activities of daily living

- C: ROM

- D: Strength

It is often used to evaluate treatment progress and to compare results of clinical trials for several specific shoulder disorders.

Based on the score, grading of the shoulder function is done as below.

- Score>30: poor function

- Score 21 to 30: fair

- Score 11 to 20: good

- Score <11: excellent

\section{RESULTS}

Most of the patients fell in two age groups, one being 31 to 50 years $(63.4 \%)$ and the other being $>60$ years $(20 \%)$. This trend was found to be consistent across the genders. Majority of the study population were male (76.7\%) and this complies with the literature. By occupation farmers (40\%), household (23.3\%), and daily wage workers (10\%) constituted the major chunk of the study population; 16 had pain in right shoulder and 14 in left shoulder; 2 patients had mild pain, 21 had moderate pain, and 7 had severe pain; 29 patients had some restriction of motion; 7 had history of some injury to shoulder; and only 2 patients had history of similar complaints in past. Duration of symptoms ranged from 15 days to 18 months. Patients were followed up regularly at frequent intervals. At each visit CMS was used to assess shoulder function. About 30 patients were available for analysis at 3 months. Some patients lost to follow-up and 15 patients were available for analysis at 17 months (Tables 1 and 2).

In the present study, shoulder function was assessed by CMS. Mean score at baseline was $33.87 \pm 5.91$. After 1 and 3 months, significant reduction in CMS was found with mean score of $27.80 \pm 5.17$ at 1 month $(p<0.0001)$ and $21.93 \pm 6.17$ at 3 months $(p<0.0001)$. There was significant difference in score at 1 and 3 months ( $<<0.0001)$. At 6, 12, and 17 months, the CMS was still significantly low but there was no difference in scores when compared with scores at 3 months.

Grade-wise, $63.3 \%$ cases had poor and $36.6 \%$ had fair shoulder function at baseline. This gradually improved at 1 month with $63.3 \%$ patients having fair function, and $3.34 \%$ patients having good function of affected shoulder. At 3 months, there was further improvement and we found $33.3 \%$ patients with good function, and 56.67\% patients with fair function of shoulder. One patient had

Table 1: Constant-Murley score in study population

\begin{tabular}{lllllll}
\hline & \multicolumn{5}{c}{ Timeline (months) } \\
\cline { 2 - 7 } CMS & $0(n=30)$ & $1(n=30)$ & $3(n=30)$ & $6(n=22)$ & $12(n=18)$ & $17(n=15)$ \\
\hline Mean \pm standard deviation & $33.87 \pm 5.91$ & $27.80 \pm 5.17^{*}$ & $21.93 \pm 6.17^{*, * *}$ & $22.13 \pm 5.48^{*, \#}$ & $21.94 \pm 5.36^{*, !}$ & $21.13 \pm 5.81^{*, \$}$ \\
Difference from baseline & - & 6.07 & 11.94 & 11.73 & 11.92 & 12.73 \\
\hline *
\end{tabular}

${ }^{*} p<0.0001$ compared with baseline by paired sample t-test; ${ }^{* *} p<0.0001$ compared with CMS at 1 month; ${ }^{*} p=0.277, ! p=0.613$,

$\$ p=404$ compared with CMS at 3 months

Table 2: Shoulder function assessed by CMS grading

\begin{tabular}{llclll}
\hline & & \multicolumn{4}{c}{ CMS score (grade) $[n(\%)]$} \\
\cline { 2 - 5 } Visits & $N$ & $>30$ (poor) & $21-30$ (fair) & $11-20$ (good) & $<11$ (excellent) \\
\hline Baseline & 30 & $19(63.33)$ & $11(36.67)$ & - & - \\
1 month & 30 & $10(33.33)$ & $19(63.33)$ & $1(3.34)$ & - \\
3 months & 30 & $2(6.67)$ & $17(56.67)$ & $10(33.33)$ & $1(3.33)$ \\
6 months & 22 & $2(9.09)$ & $13(59.09)$ & $7(31.82)$ & - \\
12 months & 18 & $1(5.56)$ & $10(55.55)$ & $7(38.89)$ & - \\
17 months & 15 & $1(6.66)$ & $7(46.67)$ & $6(40.00)$ & $1(6.67)$ \\
\hline
\end{tabular}


excellent functional recovery at 3 months. At 6 months, only 22 patients were evaluated on follow-up; $9.09 \%$ had poor function, whereas percentage of those with fair function increased marginally (59.09\%) compared with that at 3 months. Further at 12 and 17 months, only 18 and 15 patients were evaluated at follow-up. Most cases at 12 and 17 months had fair function. Marginal increase in percentage of patients with good function at 12 and 17 months was seen. One patient had excellent recovery at 17 months.

\section{DISCUSSION}

In our study, although mean age of the affected females was higher, statistical significance was not achieved. Lower mean age in our population suggests that younger patients are involved in physical job, which necessitates frequent overhead use of shoulder. This is further supported by finding that males $(76.7 \%)$ were frequently affected.

Pain was the most common presenting symptom. Severity of pain ranged from mild in $6.7 \%$ and moderate in $70 \%$ to severe in $23.3 \%$ patients. Pain was also associated with restriction of movements at shoulder. Shoulder pain was of 2 to 4 weeks (46.7\%) in majority of the patients, whereas $40 \%$ had pain from more than 4 weeks duration. Duration in our study ranged from 15 days to 18.3 months.

Majority of the general population had right-side dominance as was seen in our study, with $90 \%$ patients with right dominance. But in contrast to this, side of the affected shoulder did not vary in patients. Left (46.7\%) as well as right shoulder (53.3\%) was affected. Literature suggests that though tendinopathy is associated with frequent use of arm above the shoulder level, it often occurs in nondominant and in nonmanual workers.

History of similar past complaints was noted in 6.7\% patients, suggesting long-standing and recurrent tendonitis at shoulder. Along with pain and restriction of movements, wasting is sometime observed. Active movements are usually restrictive and painful but passive movements are full, albeit painful. Nontraumatic (76.7\%) etiology was the most common in our study, whereas trauma was involved in $23.3 \%$ cases. Intrinsic degeneration is the responsible mechanism for nontraumatic tendinopathy.

Diagnosis of supraspinatus tendinitis can be achieved with various investigational methods like USG, MRI, and arthroscopy. From systematic reviews of the literature, pooled sensitivities and specificities are 0.95 and 0.96 respectively. We also employed USG for diagnosis of tendinopathy detection in our study.

Magnetic resonance imaging has been used to grade cuff tendinopathy, and MRI reliability has been assessed between multiple observers., ${ }^{6,7}$ As per the literature, most patients are diagnosed by MRI; however, economic constraints prevented us from doing MRI on all patients. Hence, MRI in our study was employed only in $10 \%$ of the patients. The scoring system developed by Constant and Murley (1987) has been employed in this study.

Mean score at baseline before injection of PRP was $33.87 \pm 5.91$, with two-third study patients having poor score and one-third with fair score. At 1-month followup, mean score was significantly reduced to $27.80 \pm 5.17$ (difference from baseline: 6.07, $\mathrm{p}<0.0001$ compared with baseline). Further follow-up at 3 months revealed significant decrease in CMS (difference from baseline: 11.94, $\mathrm{p}<0.0001)$. This finding was further supported by the observation that $63.33 \%$ patients had fair shoulder function and $3.34 \%$ had good functionality at 1 month. At 3 months, one-third patients had good function at shoulder (CMS 11-20), most had fair functionality $(56.67 \%)$, whereas one patient had excellent recovery of shoulder function as assessed by CMS. At 6 months, only 22 patients were evaluated on follow-up; $9.09 \%$ had poor function, whereas percentage of those with fair function increased marginally (59.09\%) compared with 3 months. Further at 12 and 18 months, only 18 and 15 patients were evaluated at follow-up. Most cases at 12 and 18 months had fair function. Marginal increase in percentage of patients with good function at 12 and 18 months was seen. One patient had excellent recovery at 18 months. Although studies assessing utility of PRP in supraspinatus tendinitis are sparse, some investigators have studied PRP in overall tendinopathies of rotator cuff. There are no complications or adverse events reported with autologous PRP injections when they were used for tendinopathy. Only adverse events reported in literature are injection-related pain (local pain and mild discomfort). We observed no side effects in our study.

Our study has certain limitations, like small sample size and no control group. Preprocedure MRI would have been better to evaluate the shoulder pathology. We may have to compare these results with local steroid injection. We have used indigenous technique for preparation of PRP, which needs to be standardized.

In a comparative study conducted by the Department and Research Institute of Rehabilitation Medicine, Seoul, South Korea, 39 patients with supraspinatus tear were divided into experimental (PRP group) and study group (dry needling group). The clinical effect of the PRP injection was found to be superior to the dry needling from 6 weeks to 6 months after initial injection $(p<0.05)$. At 6 months, the mean Shoulder Pain and Disability Index (SPADI) was $17.7 \pm 3.7$ in the PRP group vs $29.5 \pm 3.8$ in the dry needling group $(p<0.05)$. No severe adverse effects were observed in either group. ${ }^{8}$

In another study conducted by the Department of Physical Medicine and Rehabilitation, Turkish Armed 
Forces Rehabilitation Center, 40 patients with rotator cuff tears were randomized into a PRP group $(n=20)$ or placebo group $(n=20)$. Patients received an ultrasoundguided injection into the subacromial space that contained either $5 \mathrm{~mL}$ of PRP prepared from autologous venous blood or $5 \mathrm{~mL}$ of saline solution. Comparison of the patients revealed no significant difference between the groups in Western Ontario Rotatory Cuff (WORC), SPADI, and visual analog scale (VAS) scores at 1-year follow-up ( $p=0.174, p=314$, and $p=0.904$ respectively). Similar results were found at other assessment points. Within each group, the WORC, SPADI, and VAS scores showed significant improvements compared with baseline at all time points $(\mathrm{p}<0.001){ }^{9}$

In a prospective randomized controlled study conducted by Shams et al, ${ }^{10} 40$ patients with symptomatic partial rotator cuff tears were randomized into PRP group $(n=20)$ and corticosteroid group $(n=20)$. They were assessed using the American Shoulder and Elbow Surgeons Standardized Shoulder Assessment Form, the CMS, the Simple Shoulder Test (SST), and a VAS for pain. Platelet-rich plasma injections showed earlier better results as compared with corticosteroid injections, although statistically significant better results after 6 months could not be found.

\section{CONCLUSION}

We found significant improvement in shoulder function as reflected in CMS, which reduced significantly at 1 month $(p<0.0001)$ and at 3 months $(p<0.0001)$ from baseline. Also categorically, more patients reached good score of improved overall shoulder function at 3 months. We found no side effects of PRP when used for SST in the timeframe of this study. In conclusion, PRP can be considered as a conservative modality of treatment for supraspinatus tendinopathies. This finding needs to be substantiated in a large, long-term placebo-controlled randomized clinical trial. It can also be hypothesized that the PRP can be a good treatment option for the patients with supraspinatous tendinopathy who had involvement of substance of tendon and needed regeneration of the tendon. ${ }^{11}$

\section{REFERENCES}

1. Benjamin B. Shoulder series 2: supraspinatus tendinitis. Massage Bodywork 2004 Aug/Sept;105(27):104-110.

2. Maffulli N, Longo UG, Loppini M, Denaro V. Current treatment options for tendinopathy. Exp Opin Pharmacother 2010 Sep;11(13):1-10.

3. Deberardino TM, Chang WK. Supraspinatus tendonitis [cited 2015 Nov 30]. Available from http://emedicine.medscape. com/article/93095-overview.

4. Mishra A, Woodall J, Vieira A. Treatment of tendon and muscle using platelet-rich plasma. Clin Sports Med 2009 Jan;28(1):113-125.

5. Constant CR, Murley AHG. A clinical method of functional assessment of the shoulder. Clin Orthop 1987;214:160-164.

6. Magee T, Williams D. 3.0-T MRI of the supraspinatus tendon. AJR Am J Roentgenol 2006 Oct;187(4):881-886.

7. de Jesus JO, Parker L, Frangos AJ, Nazarian LN. Accuracy of MRI, MR arthrography, and ultrasound in the diagnosis of rotator cuff tears: a meta-analysis. AJR Am J Roentgenol 2009 Jun;192(6):1701-1707.

8. Rha DW, Park GY, Kim YK, Kim MT, Lee SC. Comparison of the therapeutic effects of ultrasound-guided plateletrich plasma injection and dry needling in rotator cuff disease: a randomized controlled trial. Clin Rehabil 2013 Feb;27(2):113-122.

9. Kesikburun S, Tan AK, Yilmaz B, Ya ar E, Yazicio lu K. Platelet-rich plasma injections in the treatment of chronic rotator cuff tendinopathy: a randomized controlled trial with 1-year follow-up. Am J Sports Med 2013 Nov;41(11):2609-2616.

10. Shams A, El-Sayed M, Gamal O, Ewes W. Subacromial injection of autologous platelet-rich plasma versus corticosteroid for the treatment of symptomatic partial rotator cuff tears. Eur J Orthop Surg Traumatol 2016 Dec;26(8):837-842.

11. Singh PK, Saxena NK, Khan S. Effects of platelet-rich plasma in supraspinatus tendinopathy. J Orthop Allied Sci 2015 Oct;3(2):53-54. 